\title{
MODIFICAÇÃO DE UMA BARRA DE PULVERIZAÇÃO PARA A APLICAÇÃO DE FUNGICIDA EM TRIGO VISANDO AO CONTROLE DE GIBERELA ${ }^{1}$
}

\author{
EDIVAN PANISSON ${ }^{2}$, WALTER BOLLER ${ }^{3}$, ERLEI M. REIS ${ }^{4}$, LAÉRCIO L. HOFFMANN $^{5}$
}

RESUMO: Devido ao aumento da intensidade e da freqüência de ocorrência da giberela, doença causada pelo fungo Gibberella zeae (anamorfo Fusarium graminearum), essa vem sendo apontada como uma das moléstias de maior importância na cultura do trigo no Sul do Brasil. A giberela é uma doença de infecção floral e mesmo os fungicidas sistêmicos recomendados apresentam apenas efeito protetor das anteras. Os objetivos do presente trabalho foram avaliar a eficiência de controle e os efeitos nos grãos colhidos, de diferentes pontas de pulverização (jato plano, jato plano duplo e jato cônico vazio), diferentes arranjos dos bicos na barra de aplicação e dois volumes de calda. Em dois experimentos realizados no ano de 1999, a aplicação dos tratamentos reduziu significativamente a incidência, o número de espiguetas gibereladas e a severidade da doença, aumentando o rendimento de grãos. As pontas de pulverização, seus arranjos na barra e os volumes de calda utilizados comportaram-se de maneira semelhante em todas as variáveis avaliadas. $\mathrm{O}$ incremento do rendimento de grãos obtido nas parcelas tratadas em relação à testemunha sugere que se deve recomendar a aplicação de fungicidas para o controle da giberela, utilizando pontas que gerem gotas finas a médias com volume de calda de $200 \mathrm{~L} \mathrm{ha}^{-1}$.

PALAVRAS-CHAVE: barra de pulverização, pontas de pulverização, volume de calda.

\section{FUNGICIDAL SPRAY BOOM MODIFICATION FOR CONTROLING HEAD BLIGHT IN WHEAT CROP}

SUMMARY: The increased frequency and yield losses in wheat, associated with head blight in Winter cereal crops in Southern Brazil, caused by the fungus Gibberella zeae, have turned this disease into a major problem. Gibberella zeae enters the host plants through the anthers. To avoid infection, the fungicides need to protect the anthers from the fungus, which requires improved spray techniques. The role of different spray nozzles (flat fan, twin flat fan, and hollow cone), different nozzles arrangements, and two spray volumes on head blight control and grain yield were evaluated. In two experiments conducted in the field, at the year 1999, the fungicidal applications reduced significantly the disease incidence, the number of infected spikelets, and disease severity. The grain yield increased as well. The spray nozzles, nozzle arrangements, and spray volumes did not influence disease control and grain yield. The differences in grain yield between treated and non-treated plots made chemical control worthy, despite its low efficacy. Fungicidal sprays to control head blight should use nozzles that deliver medium to fine droplets, at a volume of $200 \mathrm{~L} \mathrm{ha}^{-1}$.

KEYWORDS: spray techniques, spray nozzles, spray volume.

\footnotetext{
${ }^{1}$ Parte da dissertação de mestrado do primeiro autor, apresentada ao Programa de Pós-Graduação em Agronomia da Faculdade de Agronomia e Medicina Veterinária da Universidade de Passo Fundo, FAMV/UPF. Trabalho parcialmente financiado pela Empresa Bayer Crop Science e apresentado no XXX Conbea.

${ }^{2}$ Eng $^{\mathrm{o}} \mathrm{Agr}^{\mathrm{o}}$, Mestre em Agronomia, e-mail: mnep@terra.com.br

${ }^{3}$ Eng $^{\circ}$ Agro ${ }^{\circ}$, Doutor, Docente da FAMV/UPF, Caixa Postal 611, 99001.970, Passo Fundo - RS, Fone/Fax: (0XX54) 316.8151, e-mail: boller@upf.br

${ }^{4}$ Eng $^{\mathrm{o}} \mathrm{Agr}^{\mathrm{o}}, \mathrm{PhD}$, Docente da FAMV/UPF, Passo Fundo - RS.

${ }^{5}$ Eng $^{\mathrm{o}} \mathrm{Agr}^{\mathrm{o}}$, MS, pesquisador científico da área de Fitopatologia da Coodetec, Cascavel - PR.

Recebido pelo Conselho Editorial em: 15-10-2001

Aprovado pelo Conselho Editorial em: 12-8-2003
} 


\section{INTRODUÇÃO}

O principal agente causal da giberela em trigo é o fungo Gibberella zeae (Schw.) Petch., anamorfo Fusarium graminearum (Schwabe). Nos últimos anos, tem sido observado aumento na intensidade e na freqüência da doença em trigo (Triticum aestivum L.) na maioria das regiões onde é cultivado (MAULER-MACHNIK \& ZAHN, 1994; Mc MULLEN et al., 1997). As epidemias ocorrem esporadicamente, em geral associadas com períodos prolongados de chuva ou alta umidade durante o estádio de antese do trigo (SUTTON, 1982; REIS, 1988; PARRY et al., 1995). A giberela é uma doença de infecção floral e pode ser devastadora quando a antese coincidir com temperatura elevada e molhamento prolongado das espigas (WIESE, 1987; MC MULLEN et al., 1997).

Os danos causados pela doença são quantitativos e qualitativos (WIESE, 1987; REIS, 1988; TUITE et al., 1990; BAI \& SHANER, 1994; MAULER-MACHNIK \& ZAHN, 1994; PARRY et al., 1995; MESTERHÁZY \& BARTÓK, 1996; REIS et al., 1996a). BAI \& SHANER (1994) descrevem que, na China, em anos com epidemias severas (50 a 100\% de incidência), foram detectadas reduções no rendimento de 10 a 40\%. Já AGRIOS (1988) e SUTY \& MAULER-MACHNIK (1996) relatam que, sob condições epidêmicas, podem ser observadas reduções no rendimento de $50 \%$. No Brasil, a doença ocorre principalmente nos Estados da região Sul e os maiores danos registrados foram de 14\%, porém a média em dez anos de experimentos foi de 5,4\% (REIS et al., 1996a).

REIS (1988) e RECOMENDAÇÕES... (2000) descrevem que o controle químico da giberela deve ser recomendado para as lavouras em que as condições climáticas são favoráveis ao estabelecimento do patógeno e que se deve levar em conta a ocorrência da doença nos últimos dez anos. MOSCHINI \& FORTUGNO (1996) salientam a importância da observação das previsões climáticas no período que compreende a antese, para decidir quanto à aplicação de fungicida.

SUTY \& MAULER-MACHNIK (1996) descrevem que o complexo de patógenos causadores de giberela nos cereais são os que apresentam as maiores dificuldades de controle. As maiores dificuldades relacionadas com o controle químico são: a) esporadicidade de sua ocorrência (REIS, 1988); b) dificuldade de aplicar os fungicidas no momento correto (REIS, 1988; MAULERMACHNIK \& ZAHN, 1994; PARRY et al., 1995; MESTERHÁZY \& BARTÓK, 1996), e c) dificuldade de atingir os sítios de infecção (as anteras) com os fungicidas (REIS et al., 1996b). MAULER-MACHNIK \& ZAHN (1994) e REIS et al. (1996b) afirmam que, para o controle de giberela, são necessários fungicidas que apresentem alta eficiência e que o controle seja realizado no momento adequado, quando a quantidade máxima de anteras estão expostas. Em conseqüência dos resultados obtidos por REIS et al. (1996), sugere-se que sejam desenvolvidos equipamentos de pulverização capazes de proporcionar melhor deposição dos fungicidas sobre as anteras. MAULERMACHNIK \& ZAHN (1994) salientam que é necessário obter uma boa cobertura das espigas para obter sucesso no controle. Para isso, é necessário utilizar equipamentos apropriados que forneçam densidade e tamanho de gota adequados. Para o controle da giberela, o momento oportuno da aplicação do tratamento químico é quando as anteras estão expostas, já que mesmo os fungicidas sistêmicos terão efeito protetor (REIS et al., 1988). O fungicida deve ser aplicado quando o máximo de anteras estão expostas, o que ocorre em geral do quinto ao oitavo dia após o início da antese, podendo variar de acordo com a cultivar, época de semeadura ou condições ambientais (REIS, 1988; VARGAS et al., 2000). Para o estudo em questão, o alvo biológico são as anteras soltas ou presas (REIS et al., 1996b), necessitando-se atingi-las para que o fungicida proporcione controle.

Em testes de laboratório, os fungicidas apresentam eficiência superior a $90 \%$, porém, no campo, ela é inferior a 75\% (RECOMENDAÇÕES..., 2000), sendo essa redução atribuída à insuficiente deposição dos fungicidas nas anteras. O fungicida tebuconazole é um dos ingredientes ativos que apresenta melhor eficiência de controle (MAULER-MACHNIK \& ZAHN, 1994; REIS et al., 1996b; SUTY \& MAULER-MACHNIK,1996). 
Os objetivos deste trabalho foram avaliar efeitos de tipos de pontas de pulverização, arranjos dos bicos na barra, volume de calda em relação ao controle da giberela no campo, além de avaliar as características que afetam o rendimento e/ou a qualidade dos grãos e a viabilidade econômica da aplicação de fungicida para o controle da giberela em trigo.

\section{MATERIAL E MÉTODOS}

Dois experimentos foram conduzidos na área experimental da Faculdade de Agronomia e Medicina Veterinária da Universidade de Passo Fundo, na safra de 1999, em duas épocas de semeadura (primeira época dia 9 de julho e segunda, dia 24 de julho). A densidade de semeadura do trigo, cultivar BR-23, foi de 370 sementes viáveis por $\mathrm{m}^{2}$, e o tamanho das parcelas foi de $1,2 \mathrm{~m} \mathrm{x}$ 5,0 m. Os tratos culturais, o manejo de plantas daninhas e de pragas foram realizados de acordo com as recomendações técnicas para a cultura do trigo (RECOMENDAÇÕES..., 1999). Para o controle de oídio (Blumeria graminis Speer f. sp. tritici Em. Marchal), foi realizada uma aplicação do fungicida tebuconazole (150 $\mathrm{g}$ i a ha $\left.{ }^{-1}\right)$ no dia 31 de agosto, na primeira época de semeadura, e no dia 4 de outubro, na segunda época.

A infecção das espigas pela giberela ocorreu sob condições naturais. Os tratamentos testados para o controle da giberela foram aplicados no estádio 65 da escala fenológica de ZADOCKS et al. (1974), nos dias 8 e 18 de outubro, nas duas épocas, respectivamente. O fungicida utilizado foi o tebuconazole $200 \mathrm{CE}$, na dose de $150 \mathrm{~g}^{\mathrm{i}} \mathrm{a} \mathrm{ha}^{-1}$. Os detalhes dos tratamentos utilizados encontram-se descritos e ilustrados na Tabela 1 e na Figura 1.

TABELA 1. Composição dos tratamentos utilizados nos experimentos para o controle da giberela em trigo.

\begin{tabular}{|c|c|c|c|c|c|c|}
\hline $\begin{array}{l}\text { Trata- } \\
\text { mento }\end{array}$ & $\begin{array}{l}\text { Barra e Ângulo } \\
\text { dos Bicos }\end{array}$ & $\begin{array}{l}\text { Forma } \\
\text { do Jato }\end{array}$ & $\begin{array}{c}\text { Ponta de } \\
\text { Pulverização }\end{array}$ & $\begin{array}{c}\text { Pressão } \\
(\mathrm{kPa})\end{array}$ & $\begin{array}{l}\text { Volume } \\
\left(\mathrm{L} \mathrm{ha}^{-1}\right)\end{array}$ & $\begin{array}{l}\mathrm{DMV}^{3} \\
(\mu \mathrm{m})\end{array}$ \\
\hline 1 & Testemunha $^{*}$ & & & & & \\
\hline 2 & Tradicional $^{1}$ & Plano & XR11002 & 200 & 200 & 240 \\
\hline 3 & Tradicional & Plano duplo & $110 \mathrm{DB} 2$ & 200 & 200 & 155 \\
\hline 4 & Tradicional & Cônico vazio & JA 2 & 333 & 200 & 90 \\
\hline 5 & Modificada ${ }^{2} 0^{\circ}$ & Plano & XR11002 & 200 & 400 & 240 \\
\hline 6 & Modificada $0^{\circ}$ & Plano duplo & 110DB2 & 200 & 400 & 155 \\
\hline 7 & Modificada $0^{\circ}$ & Cônico vazio & JA 2 & 333 & 400 & 90 \\
\hline 8 & Modificada $30^{\circ}$ & Plano & XR11002 & 200 & 400 & 240 \\
\hline 9 & Modificada $30^{\circ}$ & Plano duplo & $110 \mathrm{DB} 2$ & 200 & 400 & 155 \\
\hline 10 & Modificada $30^{\circ}$ & Cônico vazio & JA 2 & 333 & 400 & 90 \\
\hline
\end{tabular}

* Testemunha sem aplicação de fungicida durante todo o ciclo da cultura do trigo

${ }^{1}$ Barra tradicional: bicos espaçados em $0,50 \mathrm{~m}$, orientados na vertical - ver Figura 1

${ }^{2}$ Barra modificada: corpos de dois bicos espaçados em $0,50 \mathrm{~m} ; 0^{\circ}$ os dois bicos orientados na vertical e $30^{\circ}$ um bico posicionado para frente e outro para trás com ângulo de $30^{\circ}$ em relação à vertical - ver Figura 1

${ }^{3}$ Diâmetro mediano volumétrico das gotas, estimado por meio de gráficos e tabelas dos fabricantes

A aplicação do fungicida foi realizada com pulverizador pressurizado com $\mathrm{CO}_{2}$. Na Figura 1 , observam-se as duas configurações de barras utilizadas para a montagem dos bicos e das pontas de pulverização. Na barra tradicional, foram montados três bicos espaçados em $0,50 \mathrm{~m}$, orientados na vertical. Na barra modificada, foram montados seis bicos, utilizando-se de dois bicos a cada $0,50 \mathrm{~m}$ de distância. Nessa barra, a utilização de corpos de bicos giratórios duplos (Código QJ8600-2-1/4 NYB Spraying Systems Co.) possibilitou que os dois bicos fossem orientados na vertical, ou formando ângulos entre si e com a vertical (tratamentos $8 ; 9$ e 10 - Tabela 1). 
a) Barra Tradicional

b) Barra Modificada

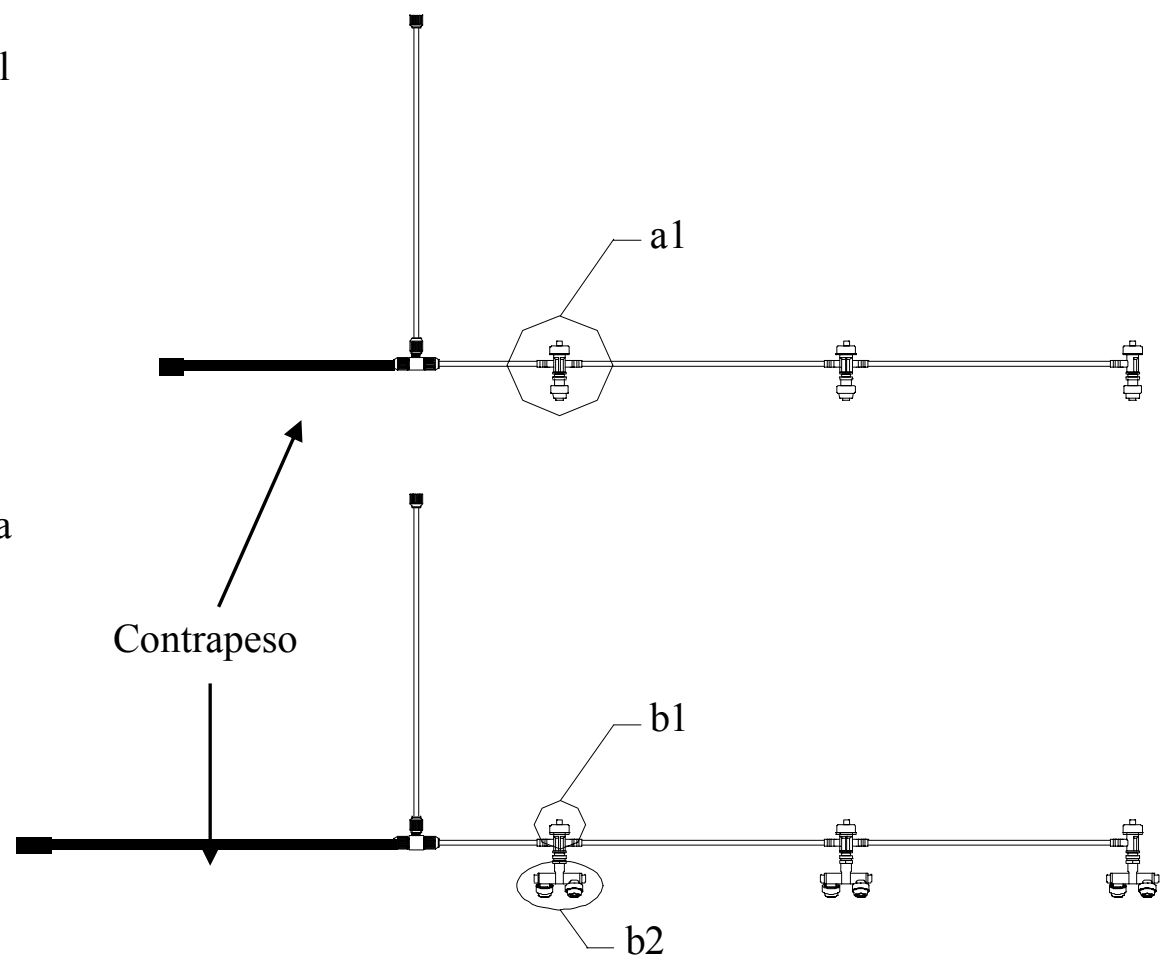

c) Detalhes
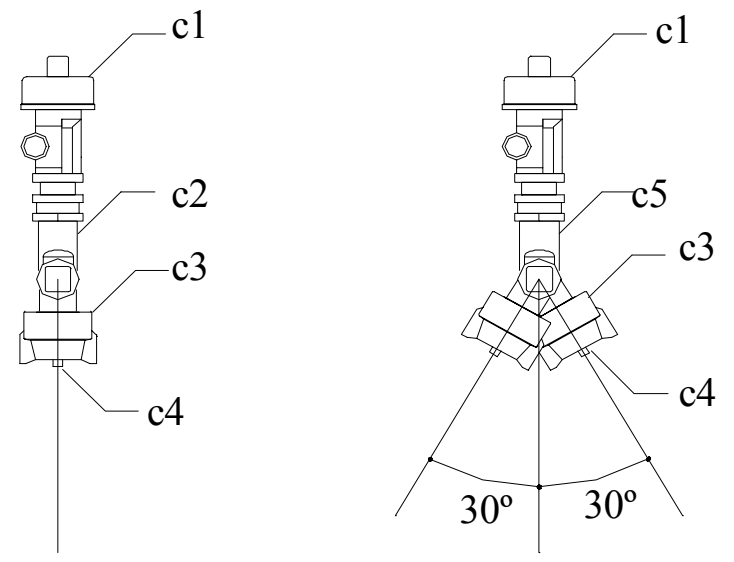

FIGURA 1. Barras utilizadas e detalhes da posição dos bicos. a) Barra tradicional, a1) corpo de bico com válvula antigotejante; b) barra modificada; b1) válvula antigotejante; b2) corpo de bico giratório duplo (Código QJ8600-2 - 1/4 NYB - Spraying Systems); c) detalhes: c1) válvula antigotejante; c2) corpo do bico; c3) capa; c4) ponta, e c5) corpo de bico giratório duplo.

A montagem dos bicos de um lado (fora do centro das barras) possibilitou realizar as pulverizações mediante deslocamento do operador ao lado das parcelas experimentais. Essa forma de condução da barra foi necessária para evitar a contaminação do operador e para não ocasionar vibrações nas plantas tratadas, que poderiam causar o escorrimento da calda recém-depositada no alvo, caso o operador se deslocasse próximo ao centro da parcela. Cabe destacar que, no momento da 
aplicação do fungicida para o controle da giberela, a cultura do trigo se encontra no máximo desenvolvimento vegetativo, dificultando o deslocamento de um operador no interior das parcelas.

A velocidade de deslocamento foi de $1 \mathrm{~m} \mathrm{~s}^{-1}$, sendo a barra conduzida a $0,4 \mathrm{~m}$ acima das espigas. No dia da aplicação, foi determinada a quantidade de anteras soltas e presas em 50 espigas coletadas ao acaso; consideraram-se anteras soltas aquelas que permanecem expostas pendentes pelo filete, e presas aquelas que permanecem aprisionadas na extremidade apical das glumas, somente com o ápice visível. Durante a aplicação, monitoraram-se a temperatura, a umidade relativa do ar e a velocidade do vento.

$\mathrm{Na}$ primeira época, a quantidade de anteras soltas/espiga foi de $18,9 \pm 8,2$ e de anteras presas/espiga de $4,2 \pm 2,2$. Durante a aplicação, as condições climáticas foram: temperatura do ar $15{ }^{\circ} \mathrm{C}$, umidade relativa do ar $74 \%$ e rajadas de vento de até $7,2 \mathrm{~km} \mathrm{~h}^{-1}$. Na segunda época, a quantidade de anteras soltas/espiga foi de $47,7 \pm 15,3$ e de anteras presas/espiga de $8,8 \pm 3,7$. Durante a aplicação, a temperatura do ar foi de $20^{\circ} \mathrm{C}$, a umidade relativa do ar $55 \%$ e a velocidade do vento inferior a $1 \mathrm{~km} \mathrm{~h}^{-1}$.

A incidência, o número de espiguetas gibereladas e a severidade foram determinados no estádio 85 (ZADOCKS et al., 1974). Após a colheita, avaliaram-se o rendimento de grãos, o peso hectolítrico, o peso de mil sementes e a incidência de $F$. graminearum em sementes. A incidência foi determinada em $0,8 \mathrm{~m}^{2}$, considerando espiga doente a que apresentasse no mínimo uma espigueta com sintoma. $\mathrm{O}$ número de espiguetas gibereladas foi determinado em 25 espigas coletadas ao acaso e convertido para porcentagem. A severidade foi calculada por meio da multiplicação da incidência pela porcentagem de espiguetas gibereladas dividindo-se por 100. A colheita foi realizada no dia 2 de dezembro de 1999, em $4,75 \mathrm{~m}^{2}$ centrais das parcelas e, para o cálculo do rendimento de grãos e do peso de mil sementes, a umidade dos grãos foi corrigida para $13 \%$.

A massa de mil sementes foi determinada em 400 sementes por parcela. Determinou-se a incidência de $F$. graminearum em 50 sementes por parcela. Após assepsia em hipoclorito de sódio, foi plaqueada em meio seletivo a Fusarium (NASH \& SNYDER, 1962) e incubada por sete dias em câmara de crescimento, iluminada com luz fluorescente sob fotoperíodo de 12 horas, com temperatura de $25 \pm 2{ }^{\circ} \mathrm{C}$.

O delineamento experimental foi de blocos casualizados, com quatro repetições por tratamento. As médias foram submetidas à análise da variância e comparadas pelo teste de Duncan, a 5\% de probabilidade. Para a análise da variância da porcentagem de controle, utilizou-se a transformação dos dados em arco-seno $\sqrt{(\mathrm{x}+0,5)}$.

\section{RESULTADOS E DISCUSSÃO}

A incidência da doença em espigas foi de $29,8 \%$, a porcentagem de espiguetas gibereladas foi de $63,5 \%$ e a severidade de $19,0 \%$, para a primeira época. Na segunda época, a incidência em espigas foi de $44,6 \%$, a porcentagem de espiguetas gibereladas $43 \%$ e a severidade de $19,3 \%$. A aplicação do fungicida reduziu significativamente a incidência e a severidade da doença nas duas épocas e na primeira apenas o número de espiguetas gibereladas.

$\mathrm{Na}$ época 1, não houve diferenças significativas do controle entre os tratamentos (Figura 2). A incidência foi a variável menos afetada, exibindo controle médio dos tratamentos de $31 \%$. Para a porcentagem de espiguetas gibereladas, o controle médio foi de $42,6 \%$ e para a severidade, de $60,3 \%$. $\mathrm{Na}$ época 2, foram observados resultados semelhantes. Os tratamentos utilizados apresentaram níveis de controle semelhantes entre si (Figura 2). O controle médio em relação à incidência foi de 44,7\%. Quanto à porcentagem de espiguetas gibereladas, não houve diferenças significativas entre a testemunha e as parcelas tratadas, sendo o controle médio de apenas $11,7 \%$. O baixo controle 
observado, provavelmente, esteja relacionado com a infecção tardia e com a ausência de efeito inibitório da colonização da espiga pelo fungicida. $\mathrm{O}$ controle, em relação à severidade, foi de $51,1 \%$, confirmando os resultados da época 1.

a

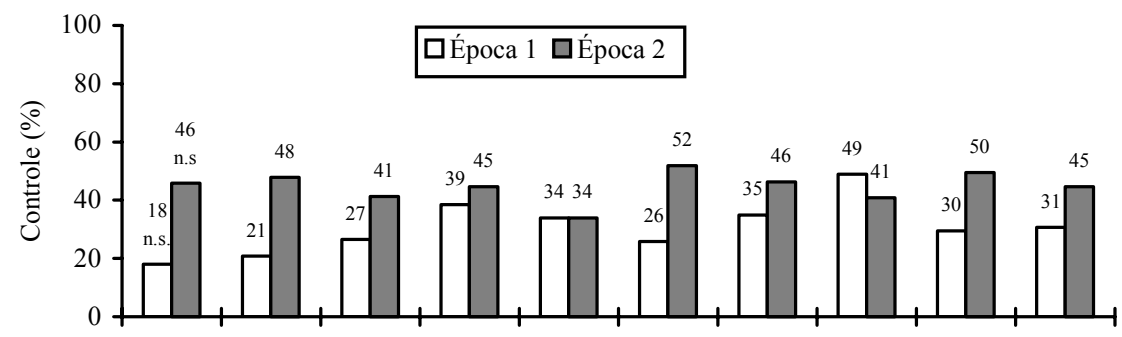

b


FIGURA 2. Efeito de pontas de pulverização, posição dessas na barra e volume de calda sobre o controle de giberela em trigo (Triticum aestivum). a) controle em relação à incidência; b) controle em relação ao número de espiguetas gibereladas; c) controle em relação à severidade. n.s. = diferenças não significativas pelo teste $\mathrm{F}$, a $5 \%$ de probabilidade.

Na época 1, a severidade na testemunha foi de $19 \%$, e o incremento do rendimento de grãos foi significativamente afetado pelos tratamentos. Em média, a aplicação de fungicidas incrementou o rendimento de grãos em $638 \mathrm{~kg} \mathrm{ha}^{-1}$ ou 17,6\%, indicando que, embora os níveis de controle da doença foram baixos, o maior efeito observado foi em relação ao rendimento de grãos. Somente os tratamentos 3 e 5 exibiram aumento significativo da massa do hectolitro. A massa de mil sementes também foi incrementada pelos tratamentos, diferindo significativamente da testemunha. A incidência do patógeno nas sementes não variou estatisticamente em relação à testemunha e apresentou média de $31,4 \%$ (Tabela 2).

$\mathrm{Na}$ época 2, o rendimento de grãos novamente foi a variável mais afetada. Os tratamentos 3 e 4 foram os que apresentaram menor rendimento de grãos, não diferindo da testemunha. Os demais tratamentos diferiram da testemunha, porém não entre si. $\mathrm{O}$ incremento médio no rendimento (Tabela 3) foi de $366 \mathrm{~kg} \mathrm{ha}^{-1}$ ou 9,7\%, inferior, portanto, ao experimento anterior. Esse menor incremento pode estar relacionado com o menor controle obtido em relação à porcentagem de espiguetas gibereladas (Figura 2), mencionado anteriormente. A infecção deve ter ocorrido mais tardiamente e, em 
conseqüência, os danos foram menores, principalmente porque os grãos já estavam formados. Nessa época, a aplicação de fungicidas não afetou o PH, o PMS e a incidência do fungo nas sementes.

TABELA 2. Efeito de pontas de pulverização, arranjo destas na barra e volume de calda sobre o rendimento de grãos $(\mathrm{RG})$, rendimento relativo $(\mathrm{RR})$, peso hectolítrico $(\mathrm{PH})$, peso de mil sementes (PMS) e incidência de Fusarium graminearum nas sementes (IS) em trigo (Triticum aestivum), obtidos na primeira época de semeadura.

\begin{tabular}{|c|c|c|c|c|c|}
\hline $\begin{array}{l}\text { Tratamentos - barra - ponta/volume de calda } \\
\qquad\left(\mathrm{L} \mathrm{ha}^{-1}\right)\end{array}$ & $\begin{array}{c}\mathrm{RG} \\
\left(\mathrm{kg} \mathrm{ha}^{-1}\right)\end{array}$ & $\begin{array}{l}\mathrm{RR} \\
(\%)\end{array}$ & $\mathrm{PH}$ & $\begin{array}{c}\text { PMS } \\
(\mathrm{g})\end{array}$ & $\begin{array}{l}\text { IS } \\
(\%)\end{array}$ \\
\hline 1 - Testemunha & $3.629 \mathrm{c}$ & - & $77,0 \mathrm{~b}$ & $35,9 \mathrm{~b}$ & 39,3 n.s. \\
\hline 2 - Tradicional - jato plano/200 & $4.348 \mathrm{a}$ & 19,8 & $78,1 \mathrm{ab}$ & $38,2 \mathrm{a}$ & 28,5 \\
\hline 3 - Tradicional - jato plano duplo/200 & $4.333 \mathrm{a}$ & 19,4 & $78,8 \mathrm{a}$ & $39,1 \mathrm{a}$ & 32,5 \\
\hline 4 - Tradicional - jato cônico vazio/200 & $4.322 \mathrm{a}$ & 19,1 & $78,1 \mathrm{ab}$ & $38,2 \mathrm{a}$ & 30,0 \\
\hline 5 - Modificada $0^{\circ}$ - jato plano $/ 400$ & $4.025 \mathrm{~b}$ & 10,9 & $78,9 \mathrm{a}$ & $38,5 \mathrm{a}$ & 32,0 \\
\hline 6 - Modificada $0^{\circ}$ - jato plano duplo/400 & $4.197 \mathrm{ab}$ & 15,7 & $78,2 \mathrm{ab}$ & $39,2 \mathrm{a}$ & 29,0 \\
\hline 7 - Modificada $0^{\circ}$ - jato cônico vazio $/ 400$ & $4.297 \mathrm{a}$ & 18,4 & $78,5 \mathrm{ab}$ & 39,0 a & 29,0 \\
\hline 8 - Modificada $30^{\circ}$ - jato plano $/ 400$ & $4.372 \mathrm{a}$ & 20,5 & $78,1 \mathrm{ab}$ & $38,7 \mathrm{a}$ & 32,5 \\
\hline 9 - Modificada $30^{\circ}$ - jato plano duplo/400 & $4.215 \mathrm{ab}$ & 16,1 & $78,0 \mathrm{ab}$ & $38,6 \mathrm{a}$ & 28,5 \\
\hline 10 - Modificada $30^{\circ}$ - jato cônico vazio/400 & $4.297 \mathrm{a}$ & 18,4 & $77,9 \mathrm{ab}$ & $39,1 \mathrm{a}$ & 32,5 \\
\hline Média & 4.204 & 17,6 & 78,2 & 38,5 & 31,4 \\
\hline C.V. $(\%)$ & 3,8 & - & 1,3 & 2,6 & 20,7 \\
\hline
\end{tabular}

* Médias seguidas da mesma letra na coluna não diferem significativamente, pelo teste de Duncan (5\%).

n.s. = diferenças não significativas pelo teste $\mathrm{F}$, a $5 \%$ de probabilidade.

TABELA 3. Efeito de pontas de pulverização, arranjo dessas na barra e volume de calda sobre o rendimento de grãos $(\mathrm{RG})$, rendimento relativo $(\mathrm{RR})$, peso hectolítrico $(\mathrm{PH})$, peso de mil sementes (PMS) e incidência de Fusarium graminearum nas sementes (IS) em trigo (Triticum aestivum), obtidos na segunda época de semeadura.

\begin{tabular}{|c|c|c|c|c|c|}
\hline $\begin{array}{l}\text { Tratamentos - barra-ponta/volume de calda } \\
\qquad\left(\mathrm{L} \mathrm{ha}^{-1}\right)\end{array}$ & $\begin{array}{c}\mathrm{RG} \\
\left(\mathrm{kg} \mathrm{ha}^{-1}\right)\end{array}$ & $\begin{array}{l}\mathrm{RR} \\
(\%)\end{array}$ & PH & $\begin{array}{c}\text { PMS } \\
(\mathrm{g})\end{array}$ & $\begin{array}{l}\text { IS } \\
(\%)\end{array}$ \\
\hline 1 - Testemunha & $3.783 \mathrm{c}$ & - & 79,8 n.s. & 34,3 n.s. & 30,5 n.s. \\
\hline 2 - Tradicional - jato plano/200 & $4.270 \mathrm{a}$ & 12,9 & 80,8 & 36,6 & 20,0 \\
\hline 3 - Tradicional - jato plano duplo/200 & $3.923 \mathrm{bc}$ & 3,7 & 79,7 & 34,8 & 23,0 \\
\hline 4 - Tradicional - jato cônico vazio/200 & $4.020 \mathrm{abc}$ & 6,3 & 80,4 & 35,3 & 21,0 \\
\hline 5 - Modificada $0^{\circ}$ - jato plano $/ 400$ & $4.119 \mathrm{ab}$ & 8,9 & 80,6 & 35,3 & 21,5 \\
\hline 6 - Modificada $0^{\circ}$ - jato plano duplo/400 & $4.156 \mathrm{ab}$ & 9,9 & 80,4 & 36,6 & 23,0 \\
\hline 7 - Modificada $0^{\circ}$ - jato cônico vazio $/ 400$ & $4.278 \mathrm{a}$ & 13,1 & 80,9 & 36,5 & 25,0 \\
\hline 8 - Modificada $30^{\circ}$ - jato plano $/ 400$ & $4.241 \mathrm{a}$ & 12,1 & 80,9 & 35,5 & 24,0 \\
\hline 9 - Modificada $30^{\circ}$ - jato plano duplo $/ 400$ & $4.110 \mathrm{ab}$ & 8,6 & 80,2 & 35,1 & 26,0 \\
\hline 10 - Modificada $30^{\circ}$ - jato cônico vazio/400 & $4.222 \mathrm{a}$ & 11,6 & 80,9 & 36,1 & 25,0 \\
\hline Média & 4.112 & 9,7 & 80,5 & 35,6 & 23,9 \\
\hline C.V. $(\%)$ & 4,4 & - & 1,0 & 4,3 & 24,8 \\
\hline
\end{tabular}

* Médias seguidas da mesma letra na coluna não diferem significativamente, pelo teste de Duncan (5\%). n.s. = diferenças não significativas pelo teste $\mathrm{F}$, a $5 \%$ de probabilidade.

REIS (1988), estudando o curso da antese em cultivares de trigo, observou que ela pode durar quatro dias em uma espiga, $12 \mathrm{em}$ uma planta e $25 \mathrm{em}$ uma lavoura. Recentemente, VARGAS et al. (2000) fizeram observações semelhantes. Por esse motivo, a desuniformidade na antese observada 
durante a aplicação dos tratamentos, mesmo em floração plena, pode estar envolvida com o baixo controle alcançado com as aplicações dos fungicidas no campo (Figura 2). Concordando com as observações feitas por Mc MULLEN (1998), as aristas podem dificultar a trajetória e a deposição do fungicida nas anteras. Por outro lado, elas também podem dificultar a deposição dos esporos sobre as anteras, dificultando a infecção.

A baixa eficiência de controle observada nos diferentes tratamentos (Figura 2) confirma os resultados obtidos por diversos pesquisadores (MARTIN \& JOHNSTON, 1982; WONG et al., 1992; MAULER-MACHNIK \& ZAHN, 1994; MESTERHÁZY \& BARTÓK, 1996; SUTY \& MAULERMACHNIK, 1996; MCMULLEN, 1998; MILUS \& WEIGHT, 1998; SCHAAFSMA \& TAMBURICILINCIC, 1998). As diferentes pontas de pulverização e os seus arranjos na barra de aplicação não proporcionaram melhor controle do que a barra tradicional com um bico posicionado na vertical, equipado com ponta de jato plano (XR-11002). Confirmam-se as observações feitas por Mc MULLEN (1998) de que a aplicação de fungicidas, utilizando diferentes bicos hidráulicos, pode proporcionar diferentes níveis de controle, mas, geralmente, não são observadas diferenças estatísticas.

Na América do Norte, a aplicação para o controle de giberela é realizada com pontas de jato plano (8002), embora não proporcione boa cobertura das espigas. Quando a mesma ponta foi utilizada com uma configuração diferente (dois bicos, um direcionado para frente e outro para trás), a cobertura foi melhor tanto nas espigas quanto em papel indicador (Mc MULLEN, 1998). Pelas observações feitas durante a aplicação dos tratamentos, a utilização dos bicos em configurações diferentes da tradicional (Tabela 1 e Figura 1), aparentemente, aumentou a cobertura das anteras, porém esse efeito não se refletiu em melhor controle da doença.

Com a utilização da barra de pulverização modificada (Figura 1), em que foram montados dois bicos a cada $0,50 \mathrm{~m}$, posicionados na vertical ou voltados para frente e para trás, o volume de calda utilizado foi duplicado (de $200 \mathrm{~L} \mathrm{ha}^{-1}$ para $400 \mathrm{~L} \mathrm{ha}^{-1}$ ). $\mathrm{O}$ aumento do volume deve ter melhorado a cobertura das espigas, porém a eficiência de controle não foi influenciada, diferindo dos resultados obtidos por SAYLER (1999), que alcançou controle superior da giberela quando utilizou volume de calda maior que $336 \mathrm{~L} \mathrm{ha}^{-1}$.

Muitas vezes, o controle obtido com a aplicação de fungicida específico para giberela não apresenta retorno satisfatório (REIS et al., 1996a; Mc MULLEN et al. 1997). Nas duas épocas, a aplicação de fungicida proporcionou incremento médio de 9,7 a 17,6\% no rendimento de grãos, confirmando os resultados obtidos por MARTIN \& JOHNSTON (1982) e WONG et al. (1992), os quais observaram que o rendimento de grãos é a variável mais afetada quando se aplica fungicida para o controle de giberela. Isto permite recomendar a aplicação de fungicida para o controle de giberela, uma vez que o retorno obtido com o incremento do rendimento de grãos é superior ao custo do fungicida, além de controlar outras doenças do trigo.

Nos últimos anos, no Brasil, foram realizados poucos estudos para verificar a viabilidade econômica da aplicação de fungicidas para o controle específico da giberela. Na safra de 1999, o custo do fungicida tebuconazole acrescido da sua aplicação situou-se em torno de U\$ 24,00. Considerandose o preço do trigo de U\$ 105,00 por tonelada, o incremento de $229 \mathrm{~kg} \mathrm{ha}^{-1}$ será equivalente ao custo do controle da giberela. Mesmo levando em conta que o ano de 1999 foi de baixa pressão de doença e que o incremento médio do rendimento de grãos em relação à testemunha sem tratamento foi de $13,65 \%$, o tratamento é economicamente viável (em uma lavoura com potencial de rendimento de $3.000 \mathrm{~kg} \mathrm{ha}^{-1} \mathrm{o}$ incremento de grãos esperado seria de 409,5 $\mathrm{kg} \mathrm{ha}^{-1}$ ). Dessa forma, a aplicação de fungicida para o controle de giberela deve ser recomendada, já que o incremento no rendimento de grãos previsto supera o custo da utilização do fungicida. Cabe considerar, ainda, o incremento da massa do hectolitro e a diminuição da incidência do fungo nos grãos que, em conseqüência, diminuirão 
os níveis de micotoxinas, ponto esse que vem merecendo mais atenção em relação à qualidade dos grãos para panificação.

\section{CONCLUSÕES}

As aplicações do fungicida tebuconazole com a barra de pulverização modificada, com diferentes pontas de pulverização e diferentes arranjos dessas na barra, não apresentam efeitos significativos sobre o controle da giberela e não aumentam o rendimento de grãos de trigo, quando comparadas com a barra tradicional equipada com pontas de jato plano XR-11002.

O equipamento tradicional utilizando pontas da série XR-11002, espaçadas em 0,50 m sobre a barra, aplicando volume de calda de $200 \mathrm{~L} \mathrm{ha}^{-1}$, mostra-se como a melhor alternativa para a aplicação do fungicida tebuconazole, visando ao controle da giberela em trigo.

O controle químico da giberela em trigo é economicamente viável e deve ser recomendado como forma de garantir a qualidade dos grãos colhidos, onde essa doença costuma ocorrer regularmente.

\section{REFERÊNCIAS BIBLIOGRÁFICAS}

AGRIOS, G.N. Plant pathology. 3.ed. St. Paul: Academic Press, 1988. 803 p.

BAI, G.; SHANER, G. Scab of wheat: prospects for control. Plant Disease, St. Paul, v.78, p.760-6, 1994.

MARTIN, R.A.; JOHNSTON, H.W. Effects and control of fusarium diseases of cereal grains in the Atlantic Provinces. Canadian Journal of Plant Pathology, Ottawa, v.4, p.210-6, 1982.

MAULER-MACHNIK, A.; ZAHN, K. Ear fusarioses in wheat - new findings on their epidemiology and control with Folicur (tebuconazole). Pflanzenschutz Nachrichten Bayer, Leverkusen, v.47, p.129$55,1994$.

Mc MULLEN, M.; JONES, R.; GALLENBERG, D. Scab of wheat and barley: a re-emerging disease of devastating impact. Plant Disease, St. Paul, v.81, p.1340-8, 1997.

Mc MULLEN, M. Fungicide technology network of the national FHB initiative - 1988. In: THE 1998 NATIONAL FUSARIUM HEAD BLIGHT FORUM, 1998, East Lansing. Proceedings... Michigan: Michigan State University, 1998. p.47-50.

MESTERHAZY, A.; BARTOK, T. Control of Fusarium head blight of weat by fungicides and its effect on the toxin contamination of the grains. Pflanzenschutz-Nachrichten Bayer, Leverkusen, v.49, p.181-98, 1996.

MILLUS, E.A.; WEIGHT, C.T. Efficacy og Quadris and Benlate applications on wheat scab in Arkansas. In: NATIONAL FUSARIUM HEAD BLIGHT FORUM, 1998, East Lansing. Proceedings... Michigan: Michigan State University, 1998. p.51-2

MOSCHINI, R.C.; FORTUGNO, C. Predicting wheat head blight incidence using models based on meteorological factors in Pergamino, Argentina. European Journal of Plant Pathology, Dordbrecht, v.102, p.211-8, 1996.

NASH, S.M.; SNYDER, W.C. Quantitative estimatives by plate counts of propagules of the bean root rot Fusarium in field soil. Phytopathology, St. Paul, v.52, p.567-72, 1962.

PARRY, D.W.; JENKINSON, P.; Mc LEOD, L. Fusarium ear blight (scab) in small grain cereals - a review. Plant Pathology, London, v.44, p.207-38, 1995. 
RECOMENDAÇÕES DA COMISSÃO SUL-BRASILEIRA DE PESQUISA DE TRIGO. In: REUNIÃO DA COMISSÃO SUL-BRASILEIRA DE PESQUISA DE TRIGO, 31., 1999, Passo Fundo: Embrapa/CNPT. 1999. 86 p.

REIS, E.M. Doenças do trigo III: giberela. 2. ed. São Paulo: E.M. REIS, 1988. 13 p.

REIS, E.M.; FERNANDES, J.M.; PICININI, E.C. Estratégias para o controle de doenças do trigo. Passo Fundo: Embrapa-CNPT. 1988. 50 p.

REIS, E.M.; BLUM, M.M.C.; CASA, R.T. Controle químico de Gibberella zeae em trigo, um problema de deposição de fungicidas em anteras. Summa Phytopathologica, São Paulo, v.22, p.39-42, 1996.

REIS, E.M.; CASA, R.T; FORCELINI, C.A. Doenças do trigo. In: KIMATI, H.; AMORIM, L.; BERGAMIN FILHO, A.; CAMARGO, L.E.A.; REZENDE, J.A.M. Manual de Fitopatologia: doenças de plantas cultivadas. 3. ed. São Paulo: Agronômica Ceres, 1997. p.725-36.

SAYLER, T. NDSU research yields more clues for improving fungicide application techniques. Disponível em: <http://www.smallgrains.org>. Acesso em: 14 jul. 1997.

SCHAAFSMA, A.W.; TAMBURIC-ILINCIC, L.; MILLER, J.D. The effect of agronomic practice on the accumulation of deoxynivalenol (DON) in winter wheat fields in Ontario, 1996-1997. In: NATIONAL FUSARIUM HEAD BLIGHT FORUM, 1998, East Lansing. Proceedings... Michigan: Michigan State University, 1998. p.7-10.

SUTTON, J.C. Epidemiology of wheat head blight and maize ear rot caused by Fusarium graminearum. Canadian Journal of Plant Pathology, Ottawa, v.4, p.195-209, 1982.

SUTY, A.; MAULER-MACHNIK A. Fusarium head blight on wheat - new findings on the epidemiology and control of Gibberella zeae the teleomorph of Fusarium graminearum with Folicur. Pflanzenschutz-Nachrichten Bayer, Leverkusen, v.49, p.55-70, 1996.

TUITE, J.; SHANER, G.; EVERSON R.J. Wheat scab in soft red winter wheat in Indiana in 1986 and its relation to some quality measurements. Plant Disease, St. Paul, v.74, p.959-62, 1990.

VARGAS, P.R.; FERNANDES, J.M.; PICININI, E.C.; HUNT, A.L. Simulação de epidemia de giberela em trigo. Fitopatologia Brasileira, Brasília, v.25, p.497-504, 2000.

WIESE, M.V. Compendium of wheat diseases. 2. ed. St. Paul: The American Phytopathological Society, 1987. $106 \mathrm{p}$.

WONG, L.S.L.; TEKAUZ, A.; LEISLE, D.; ABRAMSON, D.; Mc KENZIE, R.I.H. Prevalence, distribution and importance of fusarium headblight in wheat in Manitoba. Canadian Journal of Plant Pathology, Ottawa, v.14, p.233-8, 1992.

ZADOCKS, J.C.; CHANG, T.T.; KONZAC, C.F. A decimal code for the growth stages of cereals. Weed Research, Oxford, v.14, p.415-21, 1974. 